LA-UR-01-6198

Approved for public release; distribution is unlimited.



Los Alamos National Laboratory, an affirmative action/equal opportunity employer, is operated by the University of California for the U.S. Department of Energy under contract W-7405-ENG-36. By acceptance of this article, the publisher recognizes that the U.S. Government retains a nonexclusive, royaltyfree license to publish or reproduce the published form of this contribution, or to allow others to do so, for U.S. Government purposes. Los Alamos National Laboratory requests that the publisher identify this article as work performed under the auspices of the U.S. Department of Energy. Los Alamos National Laboratory strongly supports academic freedom and a researcher's right to publish; as an institution, however, the Laboratory does not endorse the viewpoint of a publication or guarantee its technical correctness. 


\section{MATHEMATICAL COMPARISON OF THREE TRITIUM SYSTEM EFFLUENT HTO CLEANUP SYSTEMS}

\author{
R. Scott Willms \\ Los Alamos National Laboratory \\ P. O. Box 1663 \\ Los Alamos, NM 87545, USA \\ (505) 667-5802
}

\author{
Charles Gentile and Keith Rule \\ Princeton Plasma Physics Laboratory \\ P. O. Box 451 \\ Princeton, NJ 08540 USA \\ (609) 243-2139
}

\author{
Chit Than and Philip Williams \\ Lawrence Berkeley National Laboratory \\ One Cyclotron Road \\ Berkeley, CA USA \\ (510) 486-7336
}

\section{ABSTRACT}

It important that air emissions from tritium systems be kept as low as reasonably achievable. Thus, over the years a number of gas detritiation systems have been developed. An often-used system for this purpose is one which oxidizes tritium in all forms to tritiated water followed by collection of the tritiated water on an adsorbent. These systems have been shown to be very effective at reducing tritium emissions. However, recently there has been interest in lower-cost, simpler systems which do not convert HT to the much more hazardous HTO form. One example of such a system is one based on a bubbler and dehumidifier. The bubbler collects HTO by exchange with its $\mathrm{H}_{2} \mathrm{O}$, and it humidifies the gas. The dehumidifier condenses water $\left(\mathrm{HTO}\right.$ and $\left.\mathrm{H}_{2} \mathrm{O}\right)$ from the gas and returns it to the bubbler. Besides the bubbler/dehumidifier, this paper will also consider a dehumidifier/collector and an adsorber/collector (defined later). A computer model of each configuration was written and run. The results are used to compare each system's performance. Tritium buildup in each system's liquid water, and tritium exhausted to the environment are presented and compared for all three systems.

\section{INTRODUCTION}

A number of gas detritiation systems have been developed and deployed. ${ }^{1-14}$ Often such systems oxidize tritium in all forms to tritiated water and subsequently collect the water on an adsorbent such as molecular sieve. These systems have been shown to be very effective at reducing tritium emissions.

The oxidation step described above is performed at elevated temperatures. This adds considerable cost and complexity to the system, especially at higher flowrates. This oxidation step is not necessary if much of the tritium is already in the HTO form. Also, the oxidation step converts tritium from the HT form, to the much more hazardous HTO form ( $>20,000$ times). Thus, recently there has been interest in lower-cost, simpler systems which only remove tritiated water from a gas stream.

One such system which has been deployed consists of a bubbler followed by a dehumidifier. The bubbler collects
HTO by exchange with preloaded $\mathrm{H}_{2} \mathrm{O}$, and it increases the gas humidity. The dehumidifier condenses part of the gasphase water ( $\mathrm{HTO}$ and $\mathrm{H}_{2} \mathrm{O}$ ) and returns it to the bubbler. This bubbler/dehumidifier system is shown on the lefthand-side of figure 1. It has the advantage of having very low tritium emissions during initial operation since the HTO is exchanged with $\mathrm{H}_{2} \mathrm{O}$. However, it has the disadvantage of creating more waste since untritiated water is initially loaded into the system.

A variant of this bubbler/dehumidifier system takes the same components and rearranges them into a dehumidifier/collector as shown in the middle of figure 1 . In this case the gas is fed directly to the dehumidifier, and the dehumidified gas is exhausted as previously. The dehumidifier condensate is sent to a collector tank. This system has the advantage of reducing the amount of liquid waste produced. However, tritium emissions are initially higher with this system.

A third configuration, the adsorber/collector, is shown on the right-hand-side of figure 1 . This system is very similar to the dehumidifier/collector except that the dehumidifier is replaced with an adsorber. An adsorber filled, for instance, with molecular sieve will remove humidity from a gas stream at room temperature. Periodically the adsorbent is regenerated by heating and the liberated water is transferred to the collector. Not shown on the figure is a dehumidifier or chiller capability and purge system which might be required to increase the effectiveness of the adsorber regeneration. An advantage of the adsorber/collector is that it can dehumidify gas to lower levels than the dehumidifier leading to lower tritium emissions, and it can do this with a passive system (i.e. essentially no power required since it operates at ambient conditions). Another advantage over the bubbler/dehumidifier is that the adsorber/collector produces a smaller volume of waste. The disadvantages are that the adsorber must be shutdown periodically for regeneration and that this regeneration system adds complexity and cost to the overall system. During regeneration there may be short periods of increased tritium emissions.

All three systems produce waste as liquid tritiated water. This waste form reduces the volume of waste 


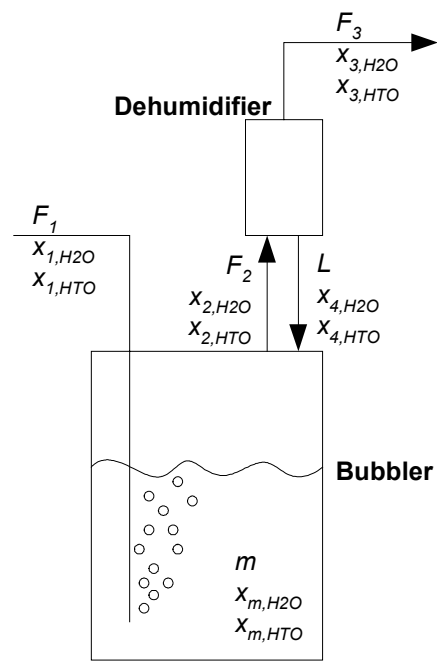

Bubbler/Dehumidifier

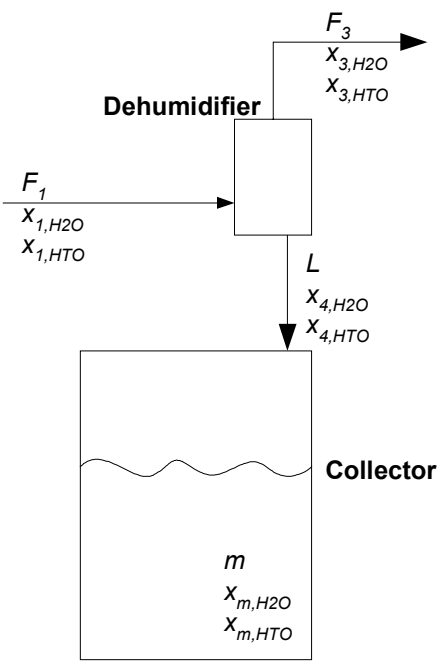

Dehumidifier/Collector

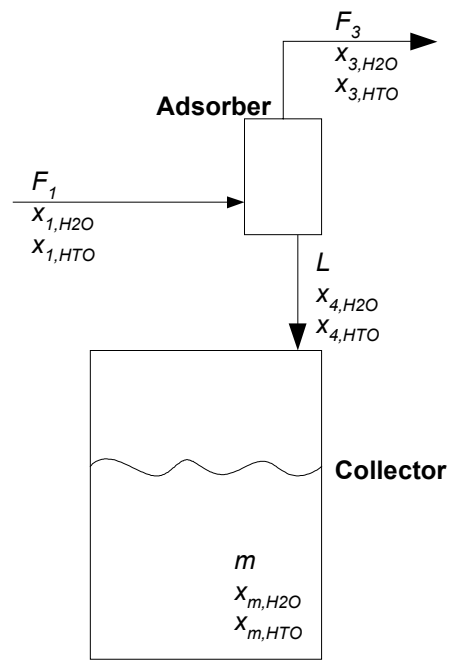

Adsorber/Collector

Figure 1 Three configurations for HTO separation from a non-condensable gas

compared to water adsorbed on molecular sieve. The liquid water can readily be solidified using one of the polyacrylate products now commercially available.

The purpose of this paper is to present a mathematical model for each of these three arrangements. Then, using practical data, the performance of each of these systems will be presented and compared. Advantages and disadvantages will be noted.

\section{MATHEMATICAL MODELS}

\section{A. Bubbler/dehumidifier}

1. Description. The flow schematic and variables used in the bubbler/dehumidifier model are shown on figure 1. A molar flowrate, $F_{l}$, is fed to the bubbler. This is labeled stream " 1 ". The feed is mostly air or inert, but has a mole fraction of $\mathrm{H}_{2} \mathrm{O}$ and HTO given as $x_{1, \mathrm{H} 2 \mathrm{O}}$ and $x_{1, \text { HTO}}$, respectively. For this model tritium in forms other than HTO (e.g. HT and tritiated methane) are neglected since such forms will not be collected in the configurations studied here. If these forms are present, their exhaust rate will be essentially equal to their inlet rate.

The feed gas is bubbled through a molar volume of water, $m$. This bubbler water is composed of $\mathrm{H}_{2} \mathrm{O}$ and HTO with mole fractions of $x_{m, \text { H2O }}$ and $x_{m, \text { НTO, }}$ respectively.

The bubbles accumulate over the water and exit the bubbler as stream "2". This stream has an overall molar flowrate, $F_{2}$, usually slightly higher than stream 1 since it picks up water from the bubbler. The water mole fractions of this stream are represented as $x_{2, \mathrm{H} 2 \mathrm{O}}$ and $x_{2, \text { HTO }}$.

Stream "2" is sent through a dehumidifier. Therein the temperature of the gas is lowered causing water to condense. The condensate is returned to the bubbler with a molar flowrate, $L$. Since this stream is composed only of water, the stream's mole fractions, $x_{4, \text { H2O }}$ and $x_{4, \text { HTO, sum }}$ to unity. The reduced-humidity gas leaves the dehumidifier with a molar flowrate of $F_{3}$. The fractions of this stream consisting of $\mathrm{H}_{2} \mathrm{O}$ and $\mathrm{HTO}$ are $x_{3, \mathrm{H} 2 \mathrm{O}}$ and

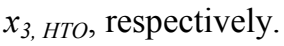

It is recognized that there is a slight difference in the pure component vapor pressure of $\mathrm{H}_{2} \mathrm{O}$ and $\mathrm{HTO}$ over a liquid mixture of these species. And, using multiple stages of separation, this effect is useful for separating these species. However, for the bubbler just described only a single stage of separation is present, and to simplify the model the isotope effects will be neglected.

2. Basic Relationships. The mole fraction of water in any stream, $i$, is given by the ratio of the water partial pressure in the stream, $p_{i, w}$, to the stream total pressure, $P_{i}$. Furthermore, the mole fraction of total water in a stream is the sum of the $\mathrm{H}_{2} \mathrm{O}$ and HTO mole fractions. These facts can be expressed as:

$$
x_{i, w}=\frac{p_{i, w}}{P_{i}}=x_{i, H 2 O}+x_{i, H T O} .
$$

The flowrate of any stream, $i$, can be expressed in terms of $F_{l}$ by noting that any flow consists of a non-water portion which is constant and a water portion which changes. This can be expressed as: 


$$
F_{i}=F_{1}-F_{1} x_{1, w}+F_{i} x_{i, w}
$$

where the first two terms on the right combine to give the non-water flowrate and the last term is the water flowrate. Solving for $F_{\mathrm{i}}$ gives:

$$
F_{i}=F_{1} \frac{\left(1-x_{1, w}\right)}{\left(1-x_{i, w}\right)}
$$

3. Water Accumulation. Water accumulation in the bubbler, assuming there is no accumulation in the dehumidifier, is given by the material balance:

$$
\frac{d m}{d t}=F_{1} x_{1, w}-F_{3} x_{3, w} .
$$

Assuming $F_{l}$ and $x_{l, w}$ are constant, the solution is:

$$
m(t)=A F_{1} t+m_{0}
$$

where:

$$
A=\frac{\left(x_{1, w}-x_{3, w}\right)}{\left(1-x_{3, w}\right)}
$$

$m(t)$ is the molar volume of water at time, $t$, and $m_{0}$ is the molar volume at time zero.

4. HTO Accumulation and Exhaust. Due to equilibration, there is a substantial change in the fraction of HTO in the water of stream 1 and the fraction of HTO in the bubbler water due to equilibration. However, beyond that point, the HTO fraction of all water remains approximately constant (neglecting isotope effects). Thus,

$$
\left(\frac{x_{m, \text { НTO }}}{x_{m, w}}\right)=\left(\frac{x_{2, \text { НTO }}}{x_{2, w}}\right)=\left(\frac{x_{3, \text { НTO }}}{x_{3, w}}\right) .
$$

Note that since the bubbler liquid water consists of water alone, $x_{m, w}=1$.

Now, again assuming there is no accumulation in the dehumidifier, the fraction of HTO in the bubbler water and in the exhaust stream can be determined by the following material balance:

$$
\frac{d\left(m x_{m, \text { HTO }}\right)}{d t}=F_{1} x_{1, \text { HTO }}-F_{3} x_{3, \text { НTO }} .
$$

For fixed $F_{l}$ and $x_{1, \text { НTO }}$ the solution is:

$$
x_{m, \text { HTO }}(t)=\frac{x_{1, H T O}}{x_{1, w}}(1-\beta(t))+x_{m, H T O, 0} \beta(t),
$$

where

$$
\beta(t)=\left(1+\frac{A F_{1} t}{m_{0}}\right)^{\frac{-x_{1, w}}{A}} \quad(A \neq 0)
$$

and $\quad \beta(t)=\exp \left(\frac{-x_{1, w} F_{1} t}{m_{0}}\right) \quad(A=0)$.

$x_{m, \text { НТО }}(t)$ is the mole fraction of HTO in the bubbler liquid water at time, $t$, and $x_{m, \text { HTO, }}$ is this same quantity at time zero. Equation (9) is invalid when $A$ is zero since a division by zero occurs. This occurs when $x_{1, w}=x_{3, w}$, i.e. when the feed and exhaust water partial pressure are the same. Since this is a case of interest, equation (10) is included which is the valid expression for $\beta(t)$ when $x_{l, w}=$ $x_{3, w}$.

With these solutions the fraction of HTO in the dehumidifier exhaust is readily calculated using equation (6).

\section{B. Dehumidifier/Collector}

1. Description. The dehumidifier/collector streams and variables are shown on figure 1. For consistency these quantities are defined identically to the bubbler/dehumidifier. As shown, however, stream " 1 " enters the dehumidifier directly. Also, stream " 2 " is no longer included since there is no flow of gas from the collector to the dehumidifier.

2. Basic Relationships. These are the same as for the bubbler/dehumidifier, so equations (1) and (2) are applied without modification to the dehumidifier/collector.

3. Water Accumulation. For the bubbler/dehumidifier, the material balance control surface for water collection was drawn around the combined bubbler and dehumidifier. The only streams crossing these surfaces were streams " 1 " and " 3 ". For the dehumidifier/collector the control surface is similarly drawn around the combined dehumidifier and collector. Again, the only streams crossing the surface are streams "1" and " 3 ". So, the water accumulation equations, equations (3), (4) and (5), remain unchanged for the dehumidifier/collector.

4. HTO Accumulation and Exhaust. This portion of the model does change. Since there is no flow through bubbler water for the dehumidifier/collector, equation (6) 
is not applicable. Rather, for streams 1, 3 and 4, there is no mechanism for changing the fraction of HTO in the stream's water (i.e. no exchange with bubbler water). Thus, the applicable relationships for these streams are:

$$
\left(\frac{x_{1, \text { HTO }}}{x_{1, w}}\right)=\left(\frac{x_{3, \text { HTO }}}{x_{3, w}}\right)=\left(\frac{x_{4, \text { HTO }}}{x_{4, w}}\right) .
$$

Since stream " 4 " is composed of water alone, $x_{4, w}=1$.

The only change to the collector originates from the dehumidifier condensate falling into the collector, so the HTO concentration in the collector is given by:

$$
\frac{d\left(m x_{m, H T O}\right)}{d t}=L x_{4, H T O}
$$

For fixed $F_{l}$ and $x_{1, \text { HTO}}$, it turns out that the solution is identical to equation (8), but the form of $\beta$ becomes:

$$
\beta(t)=\left(1+\frac{F_{1} A t}{m_{0}}\right)^{-1} \quad\left(m_{0} \neq 0\right) .
$$

The solution is only valid when $x_{1, w}>x_{3, w}$, i.e. when the humidity of the feed is greater than the output of the dehumidifier.

When the collector starts empty, i.e. when $m_{0}=0$, the solution to equation (12) becomes simply:

$$
x_{m, \text { НTO }}(t)=\frac{x_{1, \text { HTO }}}{x_{1, w}} \quad\left(m_{0}=0\right) \text {. }
$$

\section{Adsorbent/Collector}

1. Description. Comparing the adsorber/collector with the dehumidifier/collector on figure 1, it is observed that all the streams and variable definitions are identical. It is recognized that in actual operation, the adsorber/collector will be operated in a cyclic fashion. That is, when gas flows through the system (i.e. $F_{l}>0$ ), there will be no water collection (i.e. $L=0$ ). When the adsorber become saturated, it will be regenerated. During that time there will be water flow into the collector $(L>0)$ and there will be no flow through the adsorber $\left(F_{1}=0\right)$. However, on average, these details are not necessary to track. That is, over time all of the water collected in the adsorber will be transferred to the collector, so, for the purposes of this model, it is not necessary to complicate the model by keeping track of the fact that the water spent time in the adsorber.

Using this assumption the equations describing the adsorber/collector are identical to the equations for the dehumidifier/collector. The only difference will be that certain numerical values in the model, most notably the partial pressure of water in stream 3, will be different.

\section{RESULTS AND DISCUSSION}

1. Values Used for Model. The model was run using practical conditions encountered at the Princeton Plasma Physics Laboratory ${ }^{14}$. The details of this system will be the subject of a subsequent paper. ${ }^{15}$ There, a bubbler is used in combination with a dehumidifier arranged as shown in figure 1. This system was attached to the Tokamak Fusion Test Reactor vacuum vessel during decontamination and decommissioning activities. Such a system was also connected to the neutral beam enclosures. The flowrate through the bubbler/dehumidifier was 5.06 $\mathrm{mole} / \mathrm{min}$ and the bubbler was initially filled with 6311 moles of $\mathrm{H}_{2} \mathrm{O}$. The gas feed to the bubbler was $21^{\circ} \mathrm{C}$ with $40 \%$ relative humidity and the dehumidifier reduced the humidified air to a $-18^{\circ} \mathrm{C}$ dew point. Typical tritium content in the feed to the bubbler was around $3 \mathrm{mCi} / \mathrm{m}^{3}$. These conditions are summarized in Table 1.

Table 1 also lists comparable conditions for a hypothetical reconfiguration of the equipment to a dehumidifier/collector and to an adsorber/collector. For the dehumidifier/collector all of the listed conditions are the same except that the initial amount of water in the collector is zero. The same equipment, with the same properties, would be used - it would just be reconfigured. For the adsorber/collector, the conditions are the same as for the dehumidifier/collector except that the humidity exiting the adsorber is a lower $-43^{\circ} \mathrm{C}$ dew point.

Table 1 Input Values for Model Runs

\begin{tabular}{|l|l|l|l|}
\hline Parameter & Bubbler/Dehumidifier & Dehumidifier/Collector & Adsorber/Collector \\
\hline$F_{l}($ mole/min $)$ & $5.06(4 \mathrm{SCFM})$ & $5.06(4 \mathrm{SCFM})$ & $5.06(4 \mathrm{SCFM})$ \\
\hline$P_{l}, P_{3}$ (torr) & 740 & 740 & 740 \\
\hline$m_{0}$ (mole) & $6311(30 \mathrm{gal})$ & 0 & 0 \\
\hline$p_{I, w}$ (torr) & $7.5\left(21^{\circ} \mathrm{C}, 40 \% \mathrm{RH}\right)$ & $7.5\left(21^{\circ} \mathrm{C}, 40 \% \mathrm{RH}\right)$ & $7.5\left(21^{\circ} \mathrm{C}, 40 \% \mathrm{RH}\right)$ \\
\hline$P_{3, w}$ (torr) & $1.1(-18 \mathrm{DPC}, 100 \% \mathrm{RH})$ & $1.1(-18 \mathrm{DPC}, 100 \% \mathrm{RH})$ & $0.1(-43 \mathrm{DPC}, 100 \% \mathrm{RH})$ \\
\hline$x_{m, H T O, o}$ & $0.0(0.0 \mathrm{Ci} / \mathrm{L})$ & $\mathrm{N} / \mathrm{A}$ & $\mathrm{N} / \mathrm{A}$ \\
\hline$X_{I, H T O}$ & $7.8 \times 10^{-10}\left(3 \mathrm{mCi} / \mathrm{m}^{3}\right)$ & $7.8 \times 10^{-10}\left(3 \mathrm{mCi} / \mathrm{m}^{3}\right)$ & $7.8 \times 10^{-10}\left(3 \mathrm{mCi} / \mathrm{m}^{3}\right)$ \\
\hline
\end{tabular}


2. Bubbler/Dehumidifier. Model results for the bubbler/dehumidifier are given on figure 2. Plotted against time for a 12 month period are $m$ (defined above), $C_{m, \text { HTO }}$ which is the concentration of tritium as HTO in the bubbler liquid water (calculated from $x_{m, H T O}$ and presented as $\mathrm{Ci} / \mathrm{L}$ ), and $C_{3, \text { НTO }}$ which is the concentration of tritium as HTO in the gas exiting the dehumidifier (calculated from $x_{3, \text { HTO }}$ and presented as $\mathrm{mCi} / \mathrm{m}^{3}$ ). Since the dehumidifier reduces the humidity below that of the system feed, the amount of water in the bubbler increases linearly (plotted against the right hand axis). Over the course of 12 months it increases from 6311 moles (30 gal.) to 29347 (140 gal.). Both $C_{m, \text { НTO }}$ and $C_{3, \text { НTо }}$ (plotted against the left hand axis) begin at zero since the feed is exchanged with water initially containing no tritium. As tritium builds up in the bubbler water, both of these values increase - most rapidly at the beginning and more slowly as time increases. At 12 months, $C_{m, \text { НTO }}$ is $0.307 \mathrm{Ci} / \mathrm{L}$ and $C_{3, \text { HTO }}$ is $0.367 \mathrm{mCi} / \mathrm{m}^{3}$.

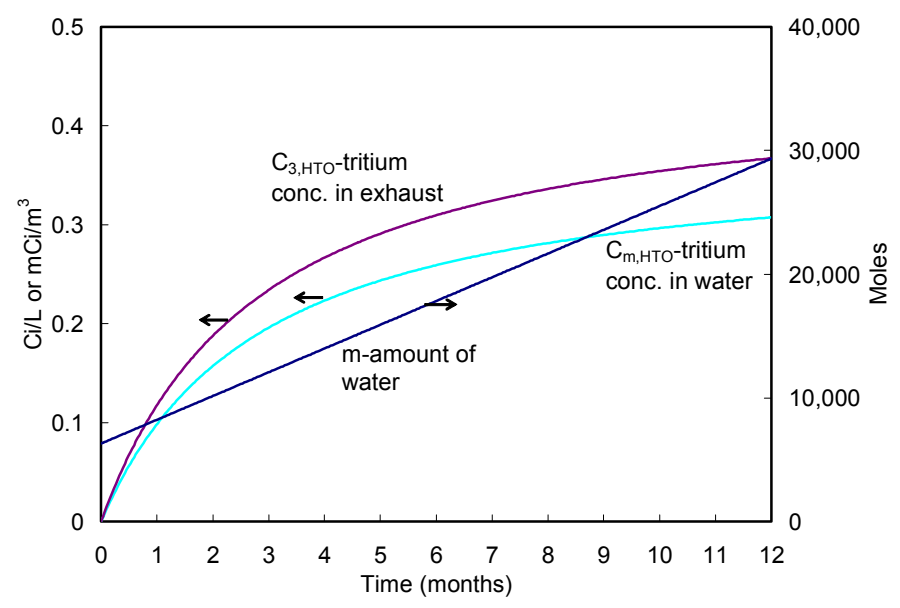

Figure 2 Results for Bubbler/Dehumidifier

3. Dehumidifier/Collector. Corresponding results for the dehumidifier/collector are given on figure 3 (solid lines) with the bubbler/dehumidifier results (dashed lines) included for comparison. As before the amount of water (now in a collector rather than a bubbler) increases linearly, but in this case the amount starts at zero. The rate of increase is identical to the bubbler/dehumidifier since the ratio of the inlet and outlet humidities are the same. Regarding concentrations, the results are quite different compared to the bubbler/dehumidifier. For the dehumidifier/collector the values are fixed at $C_{m, \text { НTO }}=$ $0.368 \mathrm{Ci} / \mathrm{L}$ and $C_{3, H T O}=0.44 \mathrm{mCi} / \mathrm{m}^{3}$. If the model is run out to much larger times, it is apparent that these are the asymptote values for the bubbler/dehumidifier. This is, of course, also apparent by inspecting the model equations.

Thus, the $\mathrm{H}_{2} \mathrm{O}$ preloaded into the bubbler serves to dilute the feed tritium and, in turn, reduce the tritium emissions. The price paid for this is an increased volume of tritiated water waste. As time increases, however, the two systems become quite similar as the tritium emissions become comparable, and the amount of tritiated water waste become comparable. These facts are quantified on figure 4 which plots the ratios $m_{\text {bubbler }} / m_{\text {dehumidifier }}$ and $C_{3, H T O, \text { dehumidifier }} / C_{3, \text { HTO, bubbler. }}$. At the end of the first day of operation, the bubbler/dehumidifier has 101 times as much liquid water, but the dehumifier/collector has a tritium emission rate that is 86 times larger. At the end of one week's operation the corresponding values are 14 and 12 , respectively, and after one month the values are 4.2 and 3.7 , respectively. Eventually both values would become unity.

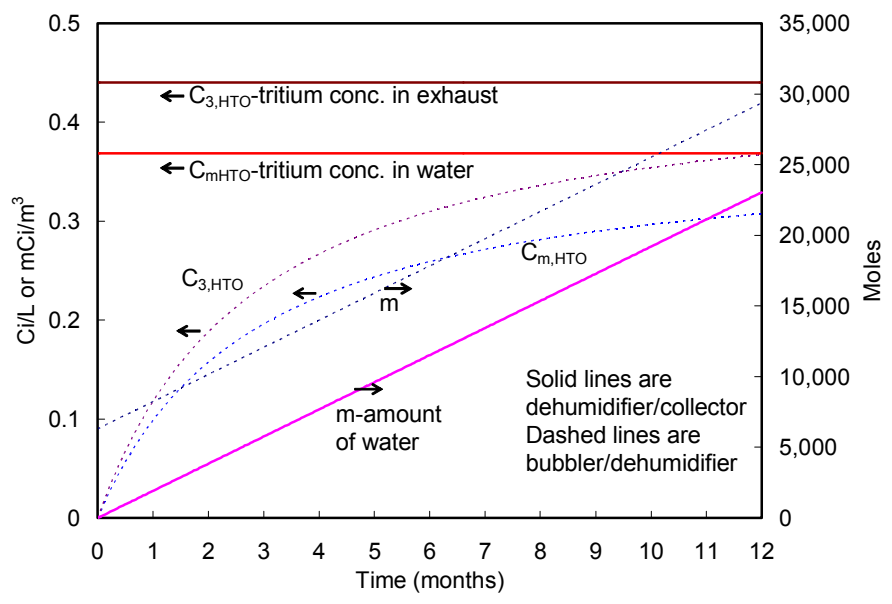

Figure 3 Results for dehumidifier/collector (with bubbler/dehumidifier for comparison)

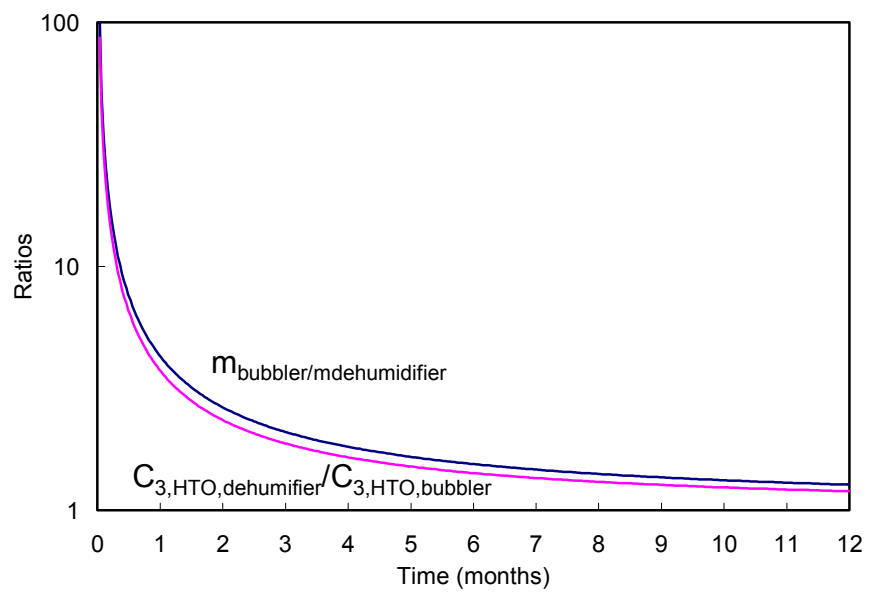

Figure 4 Comparisons of bubbler/dehumidifier results to dehumidifier/collector

4. Adsorber/Collector. The model results for the adsorber/collector are given on figure 5 (solid lines) with the bubbler/dehumidifier results (dashed lines) included for comparison. The amount of water in the collector begins at zero and increases linearly at a somewhat greater rate 
than for the bubbler since the adsorber has a lower outlet humidity resulting in more water being collected. The concentrations of HTO shown are constant with respect to time as was the case for the dehumidifier/collector. The concentration of HTO in the collector water is identical to the dehumidifier/collector at $C_{m, H T O}=0.368 \mathrm{Ci} / \mathrm{L}$ since the ratio of HTO to total water in the feed is identical in both cases. However, compared to the dehumidifier/collector, the HTO concentration in the system exhaust is much lower at $C_{3, \text { HTO }}=0.04 \mathrm{mCi} / \mathrm{m}^{3}$ because the partial pressure of total water at the adsorber exit is much lower than at the dehumidifier exit.

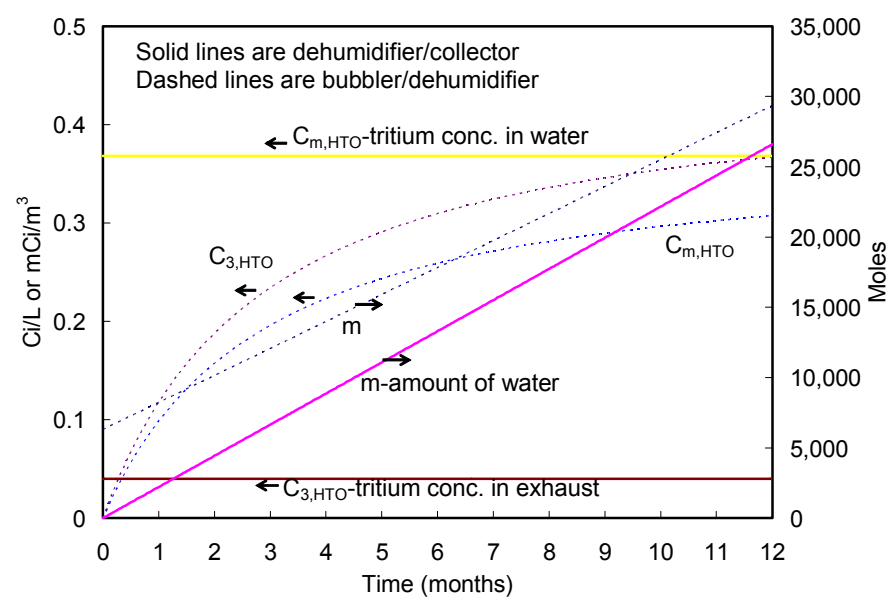

Figure 5 Results for adsorber/collector (with bubbler/dehumidifier for comparison)

As was observed previously for the dehumidifier/collector results, the adsorber/collector begins with a higher exit HTO exhaust rate and a lower amount of water than for the bubbler/dehumidifier. For the dehumidifier/collector these trade-offs continued during the entire period of operation (see figure 4). For the adsorber/collector, however, the results are quite different as shown on figure 6. During the entire 12 months of operation shown, the bubbler has more water waste compared to the collector. This curve has a shape very similar to the comparable curve on figure 4 (though the ratio will eventually become less than 1). But, quite different, is the comparison of the outlet HTO concentrations. After the first day of operation, the bubbler has an advantage with a ratio of 7.9. However, this advantage is lost at the end of the ninth day of operation when the ratio becomes 0.95 . After one month's operation the adsorber/collector has a clear advantage with the ratio being 0.34 .

5. Decontamination Factors. Another commonly used cleanup system measure of performance is the decontamination factor (DF). This is the ratio (inlet HTO concentration)/(outlet HTO concentration). These factors were calculated for all three cases and the results are summarized on figure 7 . The dehumidifier/collector and adsorber/collector have constant decontamination factors of 6.8 and 75, respectively. After day 1 the bubbler/dehumidifier has the best DF of 589. However, this value drops rapidly to become equal to the adsorber/collector during day nine. This DF continues to drop rapidly until about the two to three month point after which it slowly decays to the asymptote set by the dehumifier/collector.

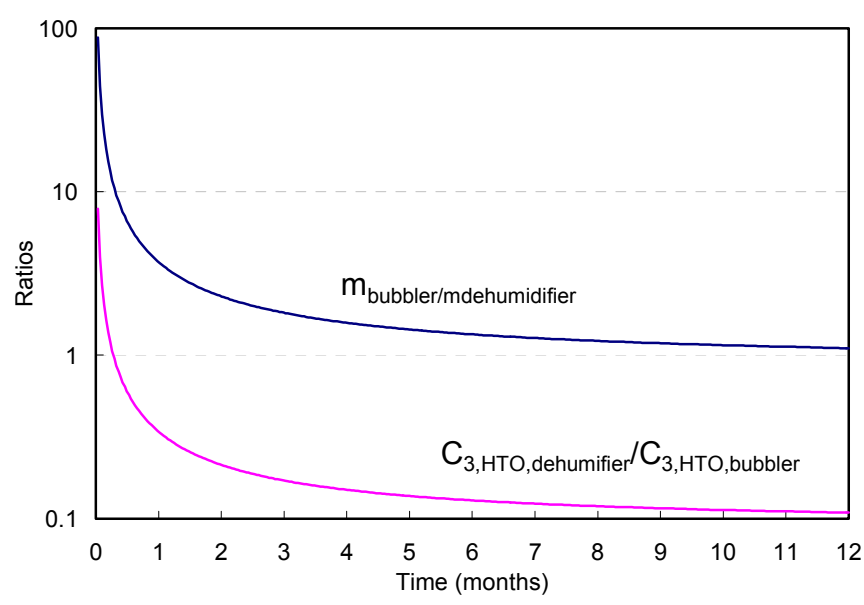

Figure 6 Comparisons of bubbler/dehumidifier results to adsorber/collector

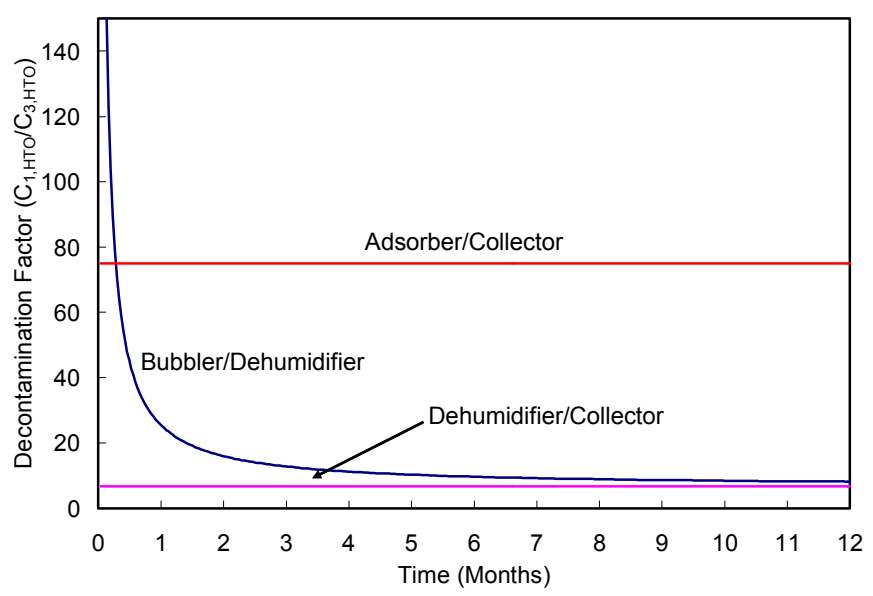

Figure 7 Decontamination factors for all three cases

6. Total Ci's and water. All of the previous HTO comparisons were presented as concentrations. It is also useful to consider performance from a total $\mathrm{Ci}$ point-ofview. Thus figure 8 is presented which shows the cumulative HTO collected as liquid water and exhausted as gas from the system in units of $\mathrm{Ci}$. The numbers at the end of each curve show the number of $\mathrm{Ci}$ at $t=12$ months. The number of $\mathrm{Ci}$ for each system sum to the number of $\mathrm{Ci}$ that entered the system over the course of 12 months, i.e. 
$179 \mathrm{Ci}$. The most dramatic difference is for the HTO exhausted as gas. The adsorber/collector is the best, only allowing 2.4 $\mathrm{Ci}$ to be released. This is followed by the bubbler/dehumidifier which releases $16.5 \mathrm{Ci}$ and finally the dehumidifier/collector which releases $26.2 \mathrm{Ci}$. The remainder of the HTO fed to the system is collected as liquid water. The adsorber/collector has the most HTO with $176.4 \mathrm{Ci}$. The bubbler/dehumidifier follows with 162.4 $\mathrm{Ci}$ and finally the dehumidifier/collector has $152.7 \mathrm{Ci}$.

It is not apparent on figure 8 , but there is a significant curvature on the early part of the results for HTO exhausted by the bubbler/dehumidifier. Initially the results are lower than those for the adsorber/collector. Then, the bubbler/dehumidifer values curve up and exceed those for the adsorber/collector after 18 days.

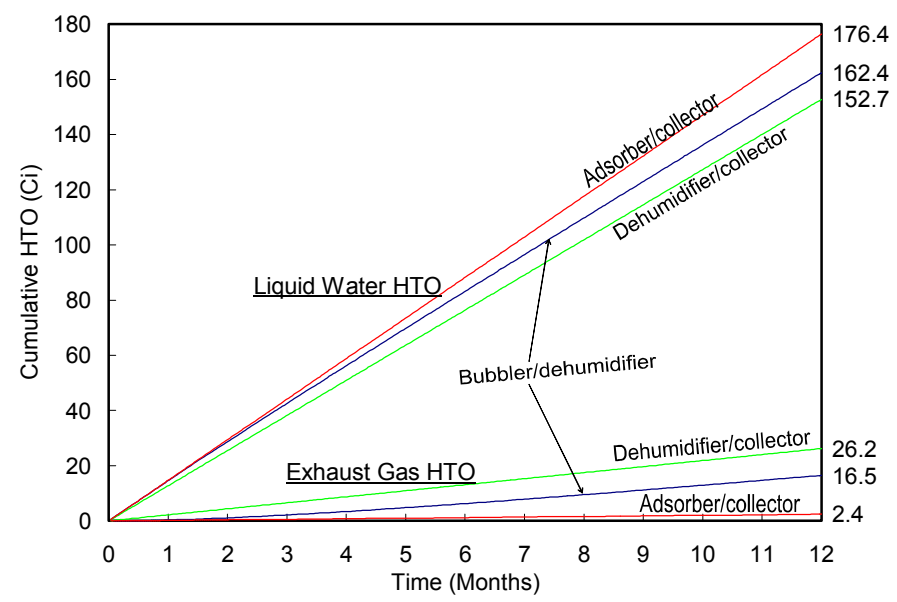

Figure 8 Cumulative HTO collected as water and released in the exhaust for all three cases

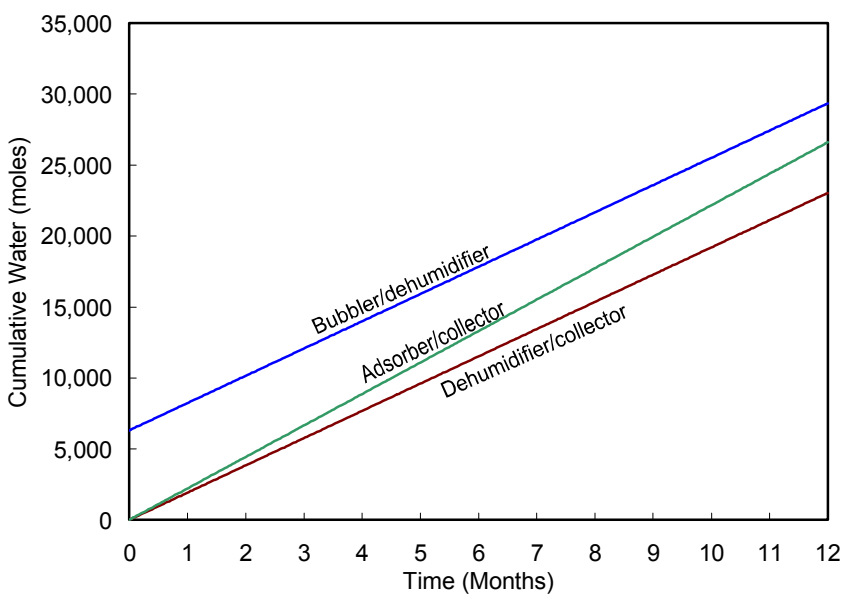

Figure 9 Quantity of liquid water waste for all three systems
One final consideration is the total volume of waste generated by each of the systems. While it was included separately on previous plots, it is useful to view the cumulative moles of liquid water waste for all three systems on a single plot, i.e. figure 9 .

\section{CONCLUSIONS}

Three systems for removing HTO from a gas stream were presented - a bubbler/dehumidifier, a dehumidifier/collector and an adsorber/collector. The mathematical equations describing each were derived and presented. These equations were used to examine a practical set of conditions. The set of conditions included a moderate humidity input and a dehumidifier that reduced this humidity to a practical value. It also assumed an adsorber that could reduce humidity to level substantially below that of the dehumidifier. Under these conditions, the results show that:

1) Initially the bubbler/dehumidifier has the lowest tritium emissions. After nine days the HTO emission rate of the adsorber/collector becomes the lowest, and after 18 days the adsorber/collector has the lowest cumulative number of Curies released.

2) The bubbler/dehumidifier initially has the largest volume of liquid waste. This condition persists until very large times of operation ( $>1$ year) when the adsorber/collector has the largest volume of waste.

3) At all times the dehumidifier/collector has the largest tritium emissions and the smallest volume of liquid waste.

4) At large times the performance of the bubbler/dehumidifier and the dehumidifier/collector become identical ( $>\sim 6$ months).

For a practical period of performance, the adsorber/collector has the lowest tritium emissions and the lowest volume of liquid waste. As such it is the most attractive system based on the results presented here. However, further considerations such as equipment availability, personnel experience, operating scenarios, local requirements, and the like may make one of the other configurations more attractive. The models developed here are useful for predicting performance and interpreting actual experience.

It is planned that follow-on work will compare these models to actual experience and will consider non-steady system feed conditions. 


\section{NOMENCLATURE}

Variables

$A$ - Collection of variable defined by equation (5)

$C_{i, j}$ - Concentration of component $j$ in stream $i\left[\mathrm{mCi} / \mathrm{m}^{3}\right]$

$C_{m, j}$ - Concentration of component $j$ in bubbler or collector water $[\mathrm{Ci} / \mathrm{L}]$

$F_{i}$ - Molar flowrate of stream $i$

$L$ - Molar flowrate of condensate into bubbler or collector

$m$ - Molar amount of water $\left(\mathrm{H}_{2} \mathrm{O}\right.$ and HTO) in bubbler or collector

$m_{0}-m$ at time zero

$p_{i, w}$ - Partial pressure of total water in stream $i$

$P_{i}$ - Total pressure of stream $i$

$t$ - Time

$x_{i, j}$ - Mole fraction of component $j$ in stream $i$

$x_{m, H T O, 0}-x_{m, \text { HTO }}$ at time zero

$x_{m, j}$ - Mole fraction of component $j$ in bubbler or collector water

$x_{i, w}$ - Mole fraction of total water in stream $i$

$\beta$ - Time dependant portion of solutions defined for various conditions by equations 9,10 and 13

Subscripts

$i$ - Subscript denoting stream number. Either 1, 2, 3 or 4 .

$j$ - Subscript denoting component. Either $\mathrm{H}_{2} \mathrm{O}$ or HTO.

$m$ - Property of the liquid water in the bubbler or collector $w$ - Total water $\left(\mathrm{H}_{2} \mathrm{O}\right.$ and $\left.\mathrm{HTO}\right)$

\section{REFERENCES}

1. R. Haange, et al., "Design of the Atmosphere Detritiation Systems for ITER," Fusion Technol., 28(3), 1491 (1995).

2. A. Aytekin and V. Corcoran, "The Design of the Gas Clean Up System for the New Tritium Facility at AWE Aldermaston," Fusion Technol., 28(3), 1463 (1995).

3. C. Talcott, et al., "Three Tritium Systems Test Assembly (TSTA) Off-Loop Experiments," Proc. of the $15^{\text {th }}$ Symposium on Fusion Engineering, October 11-15, 1993, p. 198, IEEE/NPSS, Hyannis, Massachusetts (1993).
4. J. L. Hemmerich, et al., "Installation and Inactive Commissioning of the JET Active Gas Handling System (AGHS)," Proc. of the $15^{\text {th }}$ Symposium on Fusion Engineering, October 11-15, 1993, IEEE/NPSS, Hyannis, Massachusetts (1993).

5. A. G. Heics, et al., "Design of the Tritium Scrubber System for Omega Upgrade," Fusion Technol., 28(3), 1515 (1995).

6. J. E. Klein and J. R. Wermer, "Tritium Stripping in a Nitrogen Glovebox Using Palladium/Zerolite and SAES ST 198," Fusion Technol., 28(3), 1532 (1995).

7. A. Nobile, et al., "Design Optimization of Metal Getter Reactors for Removing Tritium from Flowing Gas Streams," Fusion Technol., 28(3), 1558 (1995).

8. R. S. Willms, et al., "Comparison of Methods for Separating Small Quantities of Hydrogen Isotopes from an Inert Gas"; Proc. of the $17^{\text {th }}$ Symposium on Fusion Engineering, October 6-9, 1997, IEEE/NPSS, San Diego, California (1997).

9. D. P. Wong, J. L. Hemmerich and J. J. Monahan, "The Exhaust Detritiation System for the JET Active Gas Handling Plant-Engineering, Construction, Installation and First commissioning Results," Fusion Technol., 21(2), 572 (1992).

10. W. H. Hedley, et al., "Differences Between the TERF and the ERS Tritium Capture Systems," Fusion Technol., 21(2), 612 (1992).

11. W. T. Shmayda, et al., "Inert Gas Secondary Enclosure Clean-up System," Fusion Technol., 21(2), 616 (1992).

12. R. V. Carlson, et al., "Early Operating Experience with the Tritium Systems Test Assembly Tritium Waste Treatment System," Fusion Technol., 8(2), 2184 (1985).

13. J. E. Nasise, J. L. Anderson and Y. Naruse, "Molecular Sieve Regeneration System for Assaying HTO from Detritiation Systems," Fusion Technol., 21(3), 1974 (1992).

14. K. R. Rule, et al., "Portable Tritium Processing Using a Drum Bubbler," Plasma Devices and Operations, 6(1-3), 203 (1998).

15. R. S. Willms, et al., "Performance of Tritium System Effluent HTO Cleanup Systems and Comparison to Model," To be presented at the $19^{\text {th }}$ Symposium on Fusion Engineering, (2001). 